\title{
La importancia de la gestión y el liderazgo escolar en las instituciones de Educación Media Superior Tecnológica (CETIS y CBTIS) del estado de Tlaxcala
}

The importance of the management and school leadership in the institutions of middle and higher education technology (CETIS CBTIS) of the State of

Tlaxcala

José Margarito Rivera Badillo Universidad Popular Autónoma del Estado de Puebla, México ce.unam@hotmail.com

Judith Cavazos Arroyo Universidad Popular Autónoma del Estado de Puebla, México judith.cavazos@upaep.mx

\section{Resumen}

El presente trabajo se perfila como una contribución en el área de gestión, dirección y liderazgo escolar, atendiendo la necesidad de identificar las características de los estilos de liderazgo que aplican los directivos de Instituciones de educación media superior tecnológica (CETIS y CBTIS) del estado de Tlaxcala, México, y realizar una gestión eficaz de los planteles. Los resultados obtenidos al realizar dieciocho entrevistas a profundidad a directivos de estas instituciones, evidenciaron diferentes estilos de liderazgo privilegiándose el estilo democrático. Asimismo, con las características encontradas se establecieron las categorías que los directivos consideran aplicar en la ejecución de un estilo de liderazgo: legitimidad, motivación, enfoque en la tarea-persona, trabajo en equipo, toma de decisiones, autonomía, conocimientos y habilidades de los colaboradores para la solución de problemas, reconocimiento, competitividad para establecer estrategias de 
aprendizaje, valores, promoción de la cultura y la ética en la organización y gestión humanista.

Palabras clave: Educación Media Superior, dirección, liderazgo, gestión escolar.

\section{Abstract}

The present work is a contribution in the area of management and leadership school, in response to the need to identify the characteristics of the leadership styles that apply the managers of secondary education institutions technology (CETIS and CBTIS) of the state of Tlaxcala in Mexico, and effective management of the stocks. The resulted obtained with eighteen in-depth interviews of executives in these institutions, showed different leadership styles placing democratic styles. Also with the characteristics found categories were set that managers feel for the implementation of a leadership style: legitimacy, motivation, and focus on the task-person, team work, decision-making, autonomy, knowledge and skills of the partners for the solution of problems, recognition, and competitiveness to establish learning strategies, values, promotion of culture and ethics in the organization and management humanist.

Key words: Secondary Education, governance, leadership, school management.

Fecha Recepción: Febrero 2015 Fecha Aceptación: Julio 2015

\section{Introducción}

El presente artículo forma parte de una investigación relacionada con las características de los estilos de liderazgo y su aplicación a la gestión escolar. La discusión sobre los temas de gestión y liderazgo escolar en la búsqueda de instituciones educativas eficaces, es un factor que en la actualidad se ha privilegiado en la búsqueda científica para que un modelo educativo alcance sus metas y objetivos, de tal manera que la gestión basada en un liderazgo escolar se ha convertido en una prioridad en los modelos educativos a nivel internacional (OCDE, 2009). 
Cuando se hace referencia a la actividad educativa de la escuela, así como a la calidad de la educación, casi siempre se considera al liderazgo como uno de los elementos prioritarios; sin embargo, el liderazgo es uno de los temas que actualmente sigue en la mesa de discusión y en el campo de las aportaciones teóricas, existiendo una diferencia entre un grupo que cuenta con un líder y en otro en donde existe una ausencia de liderazgo. En este sentido, autores como Bolman y Deal (1992) y Leithwood (1994), sostienen que la actuación de los líderes afecta el ambiente y la cultura de la organización escolar, perdiéndose el sentido que los miembros de la institución escolar le otorgan a su trabajo y obstaculizando el cambio y la mejora escolar. Así, el propósito de este artículo busca identificar las características de los estilos de liderazgo de los directivos de los planteles de Educación Media Superior Tecnológica en el estado de Tlaxcala (CETIS y CBTIS).

\section{Revisión de la literatura}

Gestión Escolar. El ejercicio de la gestión escolar es una de las columnas básicas de la eficiencia organizacional de cualquier institución educativa. La gestión escolar y su relación con el liderazgo, son importantes para el desarrollo de las instituciones en lo que respecta a los aprendizajes, a sus profesores, a la currícula y sus contenidos, permitiendo que se logre la calidad educativa de las investigaciones (Alfaro, 2010). Diversos autores han redefinido el concepto de gestión escolar, coincidiendo que dicho término hace alusión al gobierno del centro escolar, la participación de los diversos sectores inmersos en el proceso educativo en la toma de decisiones y las acciones encaminadas a favorecer a los estudiantes como actores principales del proceso educativo (Cantero y Celman, 1999; Casassus, 1997; Frigerio, 2004; Sandoval, 2002).

La gestión escolar y un liderazgo eficaz podrán mejorar a la institución educativa a partir de la transformación de los recursos humanos (Pozner, 1998), liderar la gestión escolar con un enfoque no solamente transformador, sino integrador de todos los elementos que intervienen en el proceso educativo permitiendo que exista una educación de calidad en los centros escolares (SEP, 2009). La gestión escolar como un proceso que va más allá de los simples cambios administrativos, debe partir de una dimensión integral con la finalidad de contribuir al mejoramiento de la persona humana, debido a que hoy precisamente se 
necesita una educación de cualidades y extensiva a aquellos ámbitos de convivencia social (Cuenca, 2010).

Los que realicen la gestión escolar deben tener habilidades de comunicación, motivación y estimulación para formar equipos colaborativos, ejercer un estilo de liderazgo que integre todos los proyectos y buscar que la institución educativa sea eficaz (Carrillo, 2010). Para los autores de este artículo, la gestión escolar realizada a partir de un liderazgo, es un proceso dinámico que debe correlacionar los aspectos pedagógicos de la institución educativa con los espacios administrativos a partir de un enfoque humano, pero que además debe ser una forma de integrar el conocimiento, las estrategias, los esfuerzos y los recursos para garantizar una institución eficaz y con visión de futuro.

Por ello, las competencias de gestión requeridas en los directores van en relación con las tareas de la gestión escolar; de acuerdo a Garay y Uribe (2006) estas tareas son: la planificación, el fortalecimiento del liderazgo directivo, la gestión de procesos, la gestión de las competencias profesionales docentes, la orientación hacia los alumnos, sus padres y la sociedad, así como la gestión de resultados.

Las aportaciones que hacen Coronel, Carrasco y Moreno (2012) identifican los estilos de la gestión de los directores escolares con base en cuatro temas que hacen referencia a lo positivo y negativo de la gestión escolar: 1) asumir la dirección, 2) escuchar y atender al otro, 3) el saber motivar para contar con el otro y, 4) el enfoque de la gestión del director. Lograr una gestión escolar eficaz es uno de los retos que deben enfrentar los directivos de las instituciones educativas, entendida la gestión escolar como una herramienta que sistematiza las acciones dirigidas al logro de las metas y objetivos propuestos.

En el mismo sentido, los estudios de Garín y Castro (2010) sintetizan cuatro factores clave que caracterizan el tema de dirección escolar y que inciden en la calidad de la gestión de las instituciones educativas: 1) la dirección se ve implicada en todos los procesos de gestión que se producen en la escuela, 2) la dirección de los centros escolares en la actualidad debe establecerse de forma participativa, 3) la autonomía institucional debe convertirse en la garantía para el desempeño eficaz de la dirección y, 4) la dirección escolar representa una profesión poco atractiva. Una de las aportaciones interesantes realizadas sobre gestión escolar es la de Arroyo (2009), quien establece que la esencia misma de la gestión directiva 
de la educación, es la que tiene por competencia directa el proceso de toma de decisiones relacionadas con la determinación, diseño, dirección y desarrollo de lo que debe ser enseñado y aprendido por el ser social y la sociedad.

Liderazgo. Algunos de los primeros estilos de liderazgo que se establecieron fueron los propuestos por Lewin, Lippitt y White (1939), quienes propusieron la teoría que identificaba tres estilos básicos de liderazgo: autócrata, que implica un individuo con poca confianza en los miembros del grupo, cree que el dinero es la única recompensa que motiva a los trabajadores, y da órdenes sin permitir ninguna pregunta; el demócrata, que comparte la toma de decisiones con el grupo, comunica de forma objetiva y elogia a los subordinados; y el laissez-faire, que tiene poca confianza en su habilidad como líder, no establece objetivos para el grupo y minimiza la comunicación y la interacción del mismo. Hallinger y Huber (2012) comentan que las principales investigaciones realizadas sobre liderazgo escolar se han hecho en países desarrollados y anglosajones, y que solo recientemente han empezado a emerger estudios sobre liderazgo en las realidades nacionales no anglosajonas.

Como resultado de estas investigaciones se han generado diferentes conceptos sobre liderazgo escolar, por ejemplo, Marín (1998) establece que liderazgo es el desarrollo de un sistema completo de expectativas, capacidades y habilidades que permiten identificar, descubrir, utilizar, potenciar y estimular al máximo las fortalezas y la energía de todos los recursos humanos de la organización escolar. Por su parte, Alfaro (2010) sostiene que el liderazgo escolar es el arte de influir, dirigir y guiar a las personas para que potencien sus talentos, motivados en la consecución de los objetivos hacia el bien común y de la organización escolar.

Uno de los autores más reconocidos en los últimos tiempos es Leithwood (2009), quien plantea que el liderazgo es un fenómeno social que implica un propósito y una dirección, que es contextual y contingente, y es la labor de movilizar e influenciar a otros para lograr las intenciones y metas compartidas.

Al referirse al liderazgo transaccional, Bass (1985) lo describe como el proceso mediante el cual el líder reconoce lo que el seguidor desea obtener de su trabajo y procura velar porque lo obtenga siempre que su desempeño lo justifique. Asimismo se considera que los líderes 
transaccionales tienden a presentar una actitud correctiva y orientada hacia los resultados, lo cual es especialmente útil en contextos estables (Lupano y Castro, 2008). El conocido informe McKinsey da cuenta de los sistemas educativos más exitosos del mundo, revelando el rol de los directores al focalizar su función en el ejercicio del liderazgo pedagógico (Barber y Mourshead, 2007).

En la última década, tanto los informes de organismos internacionales como la producción académica en diferentes universidades e institutos de investigación sobre liderazgo, resaltan que este involucra a los líderes y liderados en una cultura común, en donde todos son responsables de contribuir al resultado colectivo (Leithwood y Louis, 2011). Cabe resaltar la observación que hace Bolívar (2013), quien destaca que el liderazgo escolar puede ser la solución a muchos de los problemas que se presentan en las instituciones educativas. Otro estilo de liderazgo es el transformador (Bass, 1985), el cual implica un proceso en el que los líderes evocan de manera permanente respuestas emocionalmente positivas de sus seguidores, además de buscar un cambio permanente en la organización. Vergara y Lazo (2011) afirman que el líder educativo debe ser un visionario que sueña con mejorar su entorno educativo, un pragmatista que evalúa con cuidado las consecuencias de sus acciones, fomentando el hábito de indagar e investigar, siendo un constante estudioso de la escolarización, de la enseñanza y del aprendizaje, con un espíritu aventurero, una actitud humilde y humana.

Por otra parte, el liderazgo distribuido (Spillane, 2006) determina que es la dirección de una institución educativa la que tiene que asumir un tipo de liderazgo, adjudicándose el rol como líder y reflejando los elementos o características que sustenten un estilo en particular. Existen otros tipos, como el liderazgo situacional (Fiedler, 1967), que estudia variables de comportamiento, orientación o motivación por la persona y orientación o motivación por la tarea; el liderazgo eficaz (Robinson, 2007) el cual está enfocado a mejorar la comprensión lectora, resolución de problemas y participación en clases de los estudiantes; y el pedagógico, en este sentido, Pont, Nusche y Moorman (2008) establecen que este tipo de liderazgo se ha convertido en una prioridad en las instituciones educativas, dado que desempeña una función determinante en la mejora de la práctica del proceso educativo en el aula, en las políticas escolares y en su relación con el contexto externo. 


\section{Metodología}

A través del uso de un enfoque metodológico cualitativo se buscó dar respuesta al objetivo planteado, para lo cual fueron considerados como parte de este proceso 18 directivos de las instituciones de educación media superior tecnológica (CETIS y CBTIS) del estado de Tlaxcala, cuatro directores, cuatro subdirectores, seis jefes de departamento y dos ex directivos. Entre estos entrevistados, ocho tenían el grado de licenciatura, ocho de maestría, uno de doctor y otro es candidato a doctor (tabla 1).

Se determinó para fines de esta investigación utilizar la entrevista a profundidad como técnica de recolección de la información y para tal efecto fue utilizada una guía de entrevista que permitió identificar las características de los estilos de liderazgo de los directivos entrevistados. Las entrevistas se realizaron en cuatro planteles de manera personalizada, garantizándole al entrevistado la confidencialidad de la información y de los resultados. Todos los entrevistados mostraron buena disposición a contestar los cuestionamientos del entrevistador. 
Tabla 1. Características de los entrevistados

\begin{tabular}{|c|c|c|c|c|c|c|c|c|}
\hline Pseudónimo & Cargo & $\begin{array}{l}\text { Antigüedad en el } \\
\text { cargo (años) }\end{array}$ & $\begin{array}{l}\text { Antigüedad } \\
\text { en educación } \\
\text { (años) }\end{array}$ & Edad & Género & Estado civil & $\begin{array}{l}\text { Lugar de } \\
\text { residencia }\end{array}$ & $\begin{array}{l}\text { Último grado de } \\
\text { estudios }\end{array}$ \\
\hline $\begin{array}{l}\text { 1.Luisa } \\
\text { López }\end{array}$ & $\begin{array}{c}\text { Jefe de } \\
\text { departamento }\end{array}$ & 2.5 & 20 & 43 & Mujer & Casada & $\begin{array}{c}\text { Ciudad de } \\
\text { Tlaxcala }\end{array}$ & $\begin{array}{c}\text { Maestría en } \\
\text { Análisis } \\
\text { Regional }\end{array}$ \\
\hline $\begin{array}{l}\text { 2.Esteban } \\
\text { Hernández }\end{array}$ & $\begin{array}{l}\text { Subdirector } \\
\text { académico }\end{array}$ & 2 & 17 & 38 & Hombre & Casado & $\begin{array}{l}\text { Apizaco, } \\
\text { Tlaxcala }\end{array}$ & $\begin{array}{l}\text { Candidato a } \\
\text { Doctor }\end{array}$ \\
\hline $\begin{array}{l}\text { 3.Carolina } \\
\text { Duarte }\end{array}$ & Director & 2.5 & 28 & 48 & Mujer & Casada & $\begin{array}{l}\text { Huamantla, } \\
\text { Tlaxcala. }\end{array}$ & Maestría \\
\hline $\begin{array}{l}\text { 4.Armando } \\
\text { Tilayatzin }\end{array}$ & $\begin{array}{c}\text { Jefe de } \\
\text { departamento }\end{array}$ & 2 & 3 & 38 & Hombre & Soltero & $\begin{array}{l}\text { Huamantla, } \\
\text { Tlaxcala }\end{array}$ & $\begin{array}{l}\text { Ingeniería } \\
\text { Industrial. }\end{array}$ \\
\hline $\begin{array}{l}\text { 5.Tomás } \\
\text { Sánchez }\end{array}$ & $\begin{array}{l}\text { Subdirector } \\
\text { académico }\end{array}$ & 2 Meses & 10 & 33 & Hombre & Casado & $\begin{array}{l}\text { Ciudad de } \\
\text { Tlaxcala }\end{array}$ & $\begin{array}{l}\text { Ingeniería } \\
\text { Mecánica }\end{array}$ \\
\hline $\begin{array}{l}\text { 6.Antonio } \\
\text { Siles }\end{array}$ & Director & 2.5 & 28 & 59 & Hombre & Casado & $\begin{array}{l}\text { Huamantla, } \\
\text { Tlaxcala }\end{array}$ & $\begin{array}{l}\text { Ingeniero } \\
\text { Industrial }\end{array}$ \\
\hline $\begin{array}{l}\text { 7.Marco } \\
\text { Antonio } \\
\text { Sánchez }\end{array}$ & Director & 5 & 12 & 38 & Hombre & Casado & $\begin{array}{l}\text { Apetatitlán, } \\
\text { Tlaxcala }\end{array}$ & Maestría \\
\hline $\begin{array}{l}\text { 8.Gerardo } \\
\text { López }\end{array}$ & $\begin{array}{c}\text { Jefe de } \\
\text { departamento }\end{array}$ & 4 & 15 & 39 & Hombre & Casado & $\begin{array}{c}\text { Amaxac de } \\
\text { Guerrero, } \\
\text { Tlaxcala. }\end{array}$ & Maestría \\
\hline $\begin{array}{l}\text { 9.Armando } \\
\text { Trápala }\end{array}$ & $\begin{array}{l}\text { Subdirector } \\
\text { académico }\end{array}$ & 3 & 14 & 40 & hombre & Casado & $\begin{array}{c}\text { San Jorge } \\
\text { Tezoquipan, } \\
\text { Tlaxcala }\end{array}$ & Licenciatura \\
\hline $\begin{array}{l}\text { 10.Antonia } \\
\text { García }\end{array}$ & $\begin{array}{c}\text { Jefe de } \\
\text { departamento }\end{array}$ & 5 & 18 & 50 & Mujer & Casada & $\begin{array}{c}\text { Ixtacuixtla, } \\
\text { Tlaxcala }\end{array}$ & Licenciatura \\
\hline $\begin{array}{l}\text { 11.Jaime } \\
\text { Martínez }\end{array}$ & $\begin{array}{l}\text { Subdirector } \\
\text { académico }\end{array}$ & 1.5 & 10 & 46 & Hombre & Casado & $\begin{array}{l}\text { Ciudad de } \\
\text { Tlaxcala, }\end{array}$ & Doctorado \\
\hline $\begin{array}{l}\text { 12. Maribel } \\
\text { Gutiérrez }\end{array}$ & $\begin{array}{c}\text { Jefe de } \\
\text { departamento }\end{array}$ & 5 & 30 & 54 & Mujer & Soltera & $\begin{array}{c}\text { Ciudad de } \\
\text { Tlaxcala }\end{array}$ & Licenciatura \\
\hline $\begin{array}{l}\text { 13.Juanita } \\
\text { Luna }\end{array}$ & $\begin{array}{c}\text { Jefe de } \\
\text { departamento }\end{array}$ & 1 & 14 & 42 & Mujer & Soltera & $\begin{array}{l}\text { San Juan } \\
\text { Totolac, } \\
\text { Tlaxcala } \\
\end{array}$ & Maestría \\
\hline $\begin{array}{l}14 . \quad \text { José } \\
\text { Dávila }\end{array}$ & Ex director & 4 & 25 & 52 & Hombre & Casado & Tlaxcala & $\begin{array}{l}\text { Licenciatura } \\
\text { en Derecho }\end{array}$ \\
\hline $\begin{array}{l}\text { 15. Juan A. } \\
\text { López }\end{array}$ & $\begin{array}{c}\text { Jefe de } \\
\text { departamento }\end{array}$ & 2 & 5 & 35 & hombre & Casado & $\begin{array}{l}\text { Ciudad de } \\
\text { Puebla. }\end{array}$ & Licenciatura \\
\hline $\begin{array}{l}\text { 16.Antonio } \\
\text { Arochi }\end{array}$ & Ex director & 1 & 33 & 57 & Hombre & Casado & $\begin{array}{l}\text { Ciudad de } \\
\text { Tlaxcala }\end{array}$ & Maestría \\
\hline $\begin{array}{l}\text { 17. Cristina } \\
\text { Rocha }\end{array}$ & $\begin{array}{c}\text { Jefa de } \\
\text { departamento }\end{array}$ & 1 & 7 & 32 & Mujer & Soltera & $\begin{array}{l}\text { Huamantla, } \\
\text { Tlaxcala }\end{array}$ & Licenciatura \\
\hline $\begin{array}{l}\text { 18. Calixto } \\
\text { Gutiérrez }\end{array}$ & $\begin{array}{c}\text { Ex directivo } \\
\text { estatal }\end{array}$ & 8 & 28 & 45 & Hombre & Casado & $\begin{array}{c}\text { San Juan } \\
\text { Totolac } \\
\text { Tlaxcala } \\
\end{array}$ & Licenciatura \\
\hline
\end{tabular}

Fuente: elaboración propia con información de los entrevistados.

\section{Resultados}

El análisis permitió identificar 13 categorías vinculadas a las características de liderazgo de los directores, las cuales son: legitimidad, motivación, enfoque en la tarea-persona, trabajo en equipo, toma de decisiones, autonomía, conocimientos y habilidades de los colaboradores para la solución de problemas, reconocimiento, competitividad para establecer estrategias de aprendizaje, valores, promoción de la cultura, la ética en la organización y gestión humanista. 


\section{Legitimidad del acceso a la dirección}

Las formas de acceder a los puestos directivos en las instituciones de Educación Media Superior Tecnológica (CETIS y CBTIS) en el estado de Tlaxcala, contribuyen a la legitimidad en la ejecución de la gestión. En esta categoría, los resultados obtenidos y que mencionaron los entrevistados fueron dos formas de hacerlo. La primera que describen los entrevistados es en relación a los directores que realizaron un examen de oposición y, la segunda forma implica que para cubrir otros puestos de dirección (por ejemplo, jefaturas de departamento y subdirección) se puede llegar por invitación del director(a). Sin embargo, actualmente ya se hace examen de oposición para estos puestos. Así, comentaron algunos de los directivos entrevistados:

"Es a través de un examen de oposición, convocatoria emitida por la Secretaría y acatar sus lineamientos" (Carolina Duarte, directora).

"Primeramente es una invitación a trabajar ahí en el puesto y, posteriormente, si uno acepta ya le dan el nombramiento" (Antonia García, jefa de departamento).

\section{Motivación de los directivos}

Los resultados obtenidos en relación a esta categoría mostraron que en la actividad laboral, los directivos en su generalidad comentaron que es importante auto-motivarse, pero también debe darse la motivación de los superiores hacia sus seguidores. Algunos comentarios son:

"La motivación depende de uno mismo, en el sentido de que si uno le pone optimismo, buena cara a las cosas, uno puede transmitir lo mismo a los compañeros" (Tomás Sánchez, subdirector académico).

"Es importante tener la motivación de nuestros superiores para de esa manera dejar ver que el trabajo se está realizando bien; asimismo, de manera personal busco también hacer las cosas bien porque de esa manera, también uno se da cuenta que está respondiendo con el trabajo que se hace" (Maribel Gutiérrez, jefa de departamento). 


\section{Enfoque en la tarea-persona}

Los resultados obtenidos en relación al enfoque en la tarea indicaron que los directivos dedican la mayor parte del tiempo a las tareas, en segundo término a las personas y, por último, al aspecto académico. En este sentido, algunos entrevistados comentaron:

"Tengo que enfocarme en las tareas, pero es necesario escuchar a las personas que tienen problemas personales, porque si te cierras generas un conflicto y el compañero se da la idea de que para qué viene a trabajar si no tiene apoyo de su jefe" (Juan A. López, jefe de departamento).

"El plantel en el que me encuentro precisamente tiene muchas necesidades y a veces hay que dedicarle también a lo que es la parte del recurso humano" (Luisa López, jefe de departamento).

\section{Trabajo en equipo}

En los resultados se observa que algunos entrevistados consideran que es fácil trabajar en equipo; pero la experiencia de otros directivos evidencia también que la antigüedad de los colaboradores y la actitud asumida pueden complicar la generación de cambios y mejoras en la institución. Algunos de los entrevistados comentaron:

"No, yo digo que no es difícil el trabajo en equipo, desde el momento en que se comparte lo que son los objetivos, el plan de trabajo, el proyecto de la institución y si la gente está enterada, sabe que va a trabajar para el bien de la escuela" (Antonio Siles, director).

"En realidad, no es difícil trabajar en equipo, ya que si nosotros hacemos un trabajo colegiado, hacemos un buen equipo”. (Esteban Hernández, subdirector académico).

"Por ejemplo, para que esta situación tenga los resultados que los padres desean ver reflejados al final, no solamente depende de los maestros, de los docentes; depende de la institución, del equipo de trabajo que forma parte la dirección y el equipo somos todos no nada más es Dirección”. (Antonia García, jefe de departamento).

\section{Toma de decisiones}


Los directivos comentan que las decisiones relevantes se toman colegiadamente, es decir, de forma colectiva. Se considera que las decisiones consensadas contribuyen a establecer una cultura de colaboración al interior de la institución, por lo que los aciertos y las consecuencias de las decisiones tomadas se asumen de forma grupal.

"La toma de decisiones se realiza de manera conjunta, atendiendo determinados indicadores y parámetros establecidos por áreas centrales, porque el trabajo colectivo nos lleva a tomar mejores decisiones" (José Dávila, ex director).

"Como lo había mencionado, creo que esto (la toma de decisiones) se hace de manera colectiva". (Esteban Hernández, subdirector académico).

\section{Autonomía}

Los resultados mostraron que existe autonomía, creatividad y libertad para desarrollar la actividad profesional como directivo y líder de una institución de Educación Media Superior Tecnológica (CETIS y CBTIS) en el estado de Tlaxcala, sin embargo, se deben apegar a la norma y a las disposiciones de las autoridades centrales. Al respecto algunos directivos mencionaron:

"Cuando yo identifico su creatividad (de los colaboradores) y esa libertad que ellos tienen, empezamos a desarrollar su creatividad en la responsabilidad que les estoy delegando, lo que hará posible que dentro de su libertad desarrollen su creatividad, con un alto nivel de autonomía" (Gerardo López, jefe de departamento).

"Se debe tomar en cuenta la creatividad del personal, ya que he observado que sus actividades las realizan con mayor libertad y gusto". (Armando Trápala, subdirector académico).

\section{Conocimientos y habilidades de los colaboradores para la solución de problemas}

La mayor parte de los directivos sostiene que sus colaboradores cuentan con los conocimientos, habilidades y experiencia en el desarrollo de sus actividades, lo que les permite tomar decisiones y resolver de manera inmediata la problemática que se presente en su área. Al respecto dos entrevistados comentaron: 
"Sí, debe uno tener confianza en el personal que colabora con uno, de esta manera ellos pueden tomar decisiones y resolver la problemática de sus áreas de trabajo sin estar considerando la presencia del superior” (José Dávila, ex director).

"Todos los colaboradores tienen los conocimientos, las capacidades y las habilidades para resolver un problema" (Armando Tilayatzin, jefe de departamento).

\section{Reconocimiento}

Se encontró que los directivos de los diferentes niveles de la estructura organizacional establecen diversas estrategias para reconocer el trabajo de sus colaboradores en el desarrollo de sus responsabilidades. Así lo expresó uno de los entrevistados:

"Pues se trata de valorar en este caso más que el cumplimento, el desempeño, el tiempo y forma de los colaboradores. Una forma de motivación es reconocer su desempeño a través de un reconocimiento, de una mención en algún evento o definitivamente de reconocérselo de manera personal mediante una felicitación" (Luisa López, jefa de departamento).

\section{Competitividad para establecer estrategias de aprendizaje}

Los resultados relacionados con esta categoría, establecieron que los directivos pondrán en práctica mecanismos para que sus colaboradores, principalmente los docentes, puedan ser competitivos y establezcan estrategias de aprendizaje con el propósito de que sus alumnos se involucren en la obtención del conocimiento. En este sentido los entrevistados comentaron:

"Establecer estrategias de competitividad aplicadas a la situación académica. Estamos en un marco de competitividad en la que tenemos que hacer propuestas innovadoras, haciendo usos de la tecnología de la información que es una de las actividades prioritarias" (Gerardo López, jefe de departamento).

"Sentimos que tenemos el equipamiento y las estrategias, capacidad y competitividad de los docentes para satisfacer las necesidades de los alumnos" (Armando Tilayatzin). 


\section{Valores}

Los valores que ponen en práctica los directivos se visualizan como claves en la ejecución del estilo de liderazgo ejercido en una institución. Al respecto algunos de los entrevistados comentaron:

"El respeto y la responsabilidad son dos valores que siempre procuro que se den en mi actividad laboral. Sabedora de que tenemos diversidad de opinión y de actuar, lo primero es respetar a las personas" (Carolina Duarte, directora).

"Los valores primero que nada deben ser personales y luego profesionales. Algunos de los más importantes son el respeto, la puntualidad, la honestidad, la legalidad y, sobre todo, el trabajo en equipo" (María Luisa López, jefe de departamento).

"Desde mi óptica, los valores que trato de reflejar con mis colaboradores son respeto, orden y disciplina, trato amable, eficiencia, eficacia, ahorro de los recursos" (Jaime Martínez, subdirector académico).

"La honestidad y la humildad, porque siendo honesto con mi trabajo se logran objetivos, y lo siguiente es ser humildes porque no llegamos a un cargo directivo sabiéndolo todo" (Calixto Gutiérrez, ex funcionario estatal).

\section{Fomento de la cultura}

Los resultados que se obtuvieron relacionados con esta categoría mostraron que la mayor parte de los entrevistados consideran que las bases para lograr un desarrollo integral de una institución educativa, son el fomento de la cultura, vinculada con las bellas artes. Algunos comentarios fueron:

"La cultura es necesaria, el arte es necesario para el desarrollo de una institución. Son cosas ineludibles ya que para comprender a una población debemos de entender su cultura" (Jaime Martínez, subdirector académico).

"Si nuestro dirigente no tiene esa visión de la ciencia, la cultura, la recreación de las actividades académicas y de la ética, yo creo que no estamos bien ubicados en lo que es la educación y cómo debería dirigirse" (Calixto Gutiérrez, ex funcionario estatal).

\section{2. Ética organizacional para el desarrollo integral}


En relación a esta categoría, los directivos entrevistados comentaron que tanto los directivos como el personal deben ejercer una ética individual y profesional.

"Nosotros, como maestros y como representantes de una institución, debemos practicar una verdadera ética, tenemos que hacer que se interesen por el medio ambiente y por los problemas sociales, entre otras cosas" (Antonio Arochi, ex director).

"Siempre he creído que tanto los valores como la ética, en particular, la ética profesional, deben ser parte de la cultura laboral, en cualquiera de sus aspectos éticos; valga la expresión, debemos estar muy imbuidos" (Jaime Martínez, subdirector académico).

\section{Gestión humanista}

Los entrevistados reconocen el valor de una gestión más humanista, la cual implica la detección de necesidades de los colaboradores, la empatía, el desarrollo y crecimiento humano. Al respecto comentaron:

"El humanismo es un aspecto importante que deben considerar los líderes ya que la situación humana nos permite conocer y saber cuáles son las necesidades de nuestros colaboradores" (Carolina Duarte, directora).

"El humanismo es fundamental, porque si no hay desarrollo humano nos estancamos, nos quedamos, vamos siendo obsoletos, todos necesitamos desenvolvernos" (Antonia García, jefe de departamento).

\section{Discusión y conclusión}

Los directivos de las instituciones de Educación Media Superior Tecnológica (CETIS y CBTIS) del estado de Tlaxcala destacan como características relevantes del liderazgo a la legitimidad, la motivación, el enfoque en la tarea-persona, el trabajo en equipo, la toma de decisiones, la autonomía, los conocimientos y las habilidades de los colaboradores para la solución de problemas, el reconocimiento, la competitividad para establecer estrategias de aprendizaje, los valores, la promoción de la cultura, la ética en la organización y la gestión 
humanista. Otros trabajos de investigación coinciden con la relevancia de algunos de estos rasgos en el perfil del líder, destacando conocimientos y habilidades, enfoque a la tareapersonas, reconocimiento, competitividad, valores, promoción de la cultura, ética de la organización y de los directivos y gestión humanista (Boyatzi y McKee, 2002, 2009; UNESCO, 2012; Schlechty, 1997; Chávez, 2013; Porter, 1998; Barba, 1997; López, 2012; Lussier y Achua, 2010; Wilber, 2000, 2007; Muñoz, 2008; Küpers 2007; Marfan, 2011 y Lorenzo, 2012).

Las características del liderazgo del directivo contribuyen a la eficiencia de la gestión en las instituciones de educación, no obstante, es necesario que el líder las practique para que pueda tener el reconocimiento de quienes día con día son parte de la institución que administra. El papel del liderazgo escolar en el desarrollo de la gestión de las instituciones educativas, así como en el establecimiento de estrategias de aprendizaje contribuye a la generación de una alta eficiencia educativa.

Debido a que muchas instituciones de educación media superior funcionan a partir de una estructura orgánica vertical en donde los directivos generan las instrucciones y sus seguidores solo las ejecutan, es necesario que se haga realidad una verdadera libertad y autonomía de los actores que participan en el acto educativo, para que la toma de decisiones permita darle solución a los problemas que se presentan en la institución.

Las características de los estilos de liderazgo de los directivos de las instituciones de educación media superior tecnológica (CETIS y CBTIS) son producto de condiciones socio históricas que se han generado a través del tiempo y que influyen en la eficiencia de su gestión escolar, por lo que es trascendente precisar el estilo de liderazgo y de gestión que desarrollan los directivos mencionados. Además deberán comprender que el liderazgo educativo constituye un elemento importante para que realicen una gestión escolar eficaz, por lo que es conveniente advertir que esa interacción habrá de lograr que los miembros de la institución educativa colaboren de manera consciente y motivada en el logro de los objetivos y metas planteadas.

En las instituciones de educación media superior tecnológica (CETIS y CBTIS) del estado de Tlaxcala, la mayoría de las funciones que realizan los directivos son de carácter 
administrativo, lo que les impide ejercer un liderazgo y una gestión adecuada de la escuela que dirigen.

Finalmente, en los tiempos actuales en los cuales se busca reformar la educación y cambiar las instituciones educativas, dos elementos fundamentales son el liderazgo educativo y la gestión escolar; es conveniente advertir que dicha conexión entre ambos elementos influye en un cambio significativo en la cultura organizacional y académica de las instituciones educativas.

\section{Bibliografía}

Alfaro, L. (2010). El componente liderazgo en la validación de un modelo de gestión escolar hacia la calidad. Educação e Pesquisa, 36 (3), 79-106.

Arroyo, J. (2009). Gestión directiva del currículum. Revista Electrónica, Actualidades Investigativas en educación, 9, 1-17.

Bass, B. M. (1981). Stogdill's, hardbook of leadership: A Survey of theory and research, Nueva York: Free Press.

Bass, B. M. (1987). Policy Implications of a new paradigm of leadership en military leadership: Translations and future trends. Conferencia desarrollada en United States Naval Academy. E.U.

Bass, B. M. \& Avolio, B. (1990). Manual for the Multifactor Leadership Questionnaire. Consulting Psychologists Press. Palo Alto, CA.

Bennis, W y Nanus, B. (1985). Leaders: The estrategies by taking charge. E.U: Harper \& Row. Publishers, N. Y.

Berne, E. (1975). Análisis Transaccional en Psicoterapia. Buenos Aires: Siglo XX.

Bolívar, A. (2010). ¿Cómo un liderazgo pedagógico y distribuido mejora los logros académicos? Internacional de investigación educativa, 3, julio-diciembre.

Bolívar, A. (2010), Liderazgo para el aprendizaje. Organización y gestión educativa, 18 (enero-febrero), 15-20. 
Bolívar, Antonio (2010). El liderazgo educativo y su papel en la mejora: una revisión actual de sus posibilidades y limitaciones. Psicoperspectivas: individuo y sociedad, 9(2), 9-33.

Bolman, C. y Deal, T. (1995) Organización y liderazgo (Delaware, Addison- Wesley. Wilmington).

Boyatzis, R. \& McKee, A. (2005). Resonant Leadership: Renewing Yourself and Connecting With Others Through Mindfulness, Hope, and Compassion. Harvard Business School Press. Boston.

Boyatzis, R. \& McKee, A. (2005). Neuroscience and Leadership: the promise of insight. Harvard Business School Press. Boston.

Bush, T. y Glover, D. (2003). School leadership: Concepts and evidence. NCSL. USA.

Cantero, G. y Celman, S. (1999). Un análisis alternativo. En SEP (ed.) Antología de gestión escolar, SEP. México.

Campbell, R., Bridges, E., Corablly, J., Nystrand R. \& Ramseyer, J. (1971). Introduction to educational administration, 4a ed. Boston: Allyn and Bacon.

Casassus, J. (1997). Marcos conceptuales para el análisis de los cambios en la gestión los sistemas educativos. En Antología de Gestión Escolar. 13-30 México.

Carriego, C. (2002). Gestionar una escuela comprometida con las demandas de su tiempo. Iberoamericana de educación. Argentina.

Carrillo, V. (2010). La gestión escolar en las instituciones educativas. Sythesis, 46, 1-12.

Cortez, (2004). Estilos de liderazgo y motivación laboral. En el ambiente educativo Revista de Ciencias Sociales (Cr). Vol. IV, núm. 106, 203-214. Costa Rica.

Drucker, P. (1992): Managing for the future, Ed. Truman Talley Books Dutton, New York.

Drucker, P. (1988): The coming of the new organization, Ed. Harvard bussiness Review, enero-febrero, 45-33. 
Freire, P. (2001). Pedagogía de la indignación. Madrid, España: Morata.

Fullan, M. (1996). Leadership for change. En K. Leihwood, J. Chapman, D. Corson, P. Hallinger y A. Hart (Eds.), "International Handbook of Educational Leadership and Administration" (701-722). Dordrecht: Kluwer Academic Publisher.

Gairín S. y Castro C. (2010) Situación actual de la dirección y gestión de los centros de enseñanza obligatoria en España. Revista española de pedagogía. Año LXVIII, n ${ }^{\circ}$ 247, septiembre-diciembre, 401-41

Kanungo, R. N. (2001). Ethical values of transactional and transformational leadership. Canadian Journal of Administrative Sciences, 18, 4, 257.

Kertész, R., Atalaya, C. I. \& Kertész V. R. (1978). Liderazgo transaccional. Como hacer que lo haga la gente. Argentina: IPPEM.

Kepner, C. (1983). El nuevo directivo racional: análisis de problemas y toma decisiones. México: Mc Graw-Hill.

Kotter, J., (1991). El directivo como líder y como ejecutivo: la simbiosis del éxito en: Harvard Business Review.

Kouzes, J. M., \& Posner, B. Z. (2007). The leadership challenge (4th ed.). San Francisco, CA: Jossey-Bass.

Küpers, W. (2007). Perspectives on Integrating Leadership and Followership. International Journal of Leadership Studies, 2, 194-221.

Küpers, W. \& Statler, M. (2008). Practically wise leadership: toward an integral understading. Culture and Organization. USA. 
Kvale, S. (1996). Interviews: An introduction to qualitave research interviewing. Thousand Oaks, CA, Sage.

Kvale, S. (2006). Interviewing between method and craft. Denmark: Department of Psychology, University of Aarhus.

Leithwood, K. \& Jatzi, D. (2006). Transformational school leadership for large. Scale reform: Effects on students, teachers, and their classroom practices. School effectiveness and school improvement, 17(2), 201-227.

Leithwood, K. Steinbach, R. Jantzi, D. (2002). School leadership and teachers motivation to implement accountability. Educational Administration Quarterly, 38, 94-119.

Leithwood, K., et al., (1996). Transformational school leadership. In: K. LEITHWOOD, et al. (eds.), International Handbok of educational leadership and administration. Dordrecht, Kluwer. 785-840.

Leithwood, K. A., et al., (1999). Changing Leadership for Changing times. BuckinghamPhiladelphia, Open University Press, E.U.

Leithwood, K. (2004). El liderazgo con éxito. El liderazgo educacional transformador en un mundo de políticas transaccionales. ICE Deusto, Actas del IV Congreso Internacional sobre Dirección de Centros Educativos, 233-245.

Longo, M. F. (2008). Liderazgo distribuido, un elemento crítico para promover la innovación. Capital humano, 226, p 84.

López, C. M. (2009). Educación Humanista Tomo 1, 2,3. Ed. Gernika. México.

López, Y. J. (2010) Liderazgo para sostener procesos de innovación en la escuela, Curriculum y formación del profesorado, 14, 1 España. 
López, C. M. y Gaeta, M.L. (2015). Ética y autorregulación en la formación de profesionales de la educación. IBERO-UPAEP. México.

López, Y. J. \& Lavié, M. J. M. (2010). Liderazgo para sostener procesos de innovación en la escuela. Profesorado. Curriculum y formación del profesorado. 72-92.

Lussier, R. y Anchua, C. (2010). Liderazgo. Teoría, aplicación y desarrollo de habilidades. Cengage Learning. México.

Marín, C. (1998, Mayo). Toma de decisiones y liderazgo. Revista Acta Académica, 22, s. p.

Martínez, A. (2006). La investigación cualitativa (síntesis conceptual). Revista IIPSI. Facultad de Psicología. (UNMSM) V. 9 No. 1. 123-146.

Maslow, A.(1972). El hombre autorrealizado, Kairós, Barcelona,

Meo, A. y Navarro, A. (1009) enseñando a hacer entrevistas, la profesión y el arte. Empíria. Revista de metodología en ciencias sociales. No. 17 pp. 123-140

Mineduc (Ministerio de Educación) (2008 a). Situación del liderazgo educativo en Chile, Santiago.

Mintzberg, H. (1991). Mintzberg y la dirección. Ed. Díaz Santos. Madrid, España.

Mogollón, A. (2006). Calidad y enfoques de la supervisión. Revista Ciencias de la Educación, 1 (23), 29-46.

Murillo, J. F. (2011). Una dirección escolar para el cambio: Del liderazgo transformacional al liderazgo distribuido. Pensamiento Imaginativo. España.

Muñoz, S. M. K. (2008) Liderazgo integral, OEvernorte.

Pont, Nusche y Moorman (2008). Mejorar el liderazgo escolar. Herramientas de trabajo. Organización para la Cooperación y el Desarrollo Económico.OCDE.

Pozner, P. (1997). El directivo como gestor de aprendizajes escolares. Buenos Aires, AIQUE. Argentina. 
Posner, Ch. (2004). "Enseñanza efectiva", Revista Mexicana de Investigación Educativa (México), vol. IX, núm. 21, 277-318.

Ramírez, L. (2o12). Calidad escolar: Un nuevo ethos escolar. Educación. 36, 51-60.

Reyes, J.L. \& Lara, R. G. (2011). El liderazgo integral en las organizaciones. Avances en Psicología Latinoamericana, 29, 1, Colombia.

Riveros-Barrera, A. (2012). La distribución del liderazgo como estrategia de mejoramiento institucional. Educación y Educadores. Vol. 15, No. 2, 289-301

Robinson, V., Lloyd, C. y Rowe, K. (2014). El impacto del liderazgo en los resultados de los estudiantes. Un análisis de los efectos diferenciales de los estudiantes. Un análisis de los efectos diferenciales de los tipos de liderazgo. Revista Iberoamericana sobre calidad, eficiencia y cambio en educación, 12, 1-16.

Rodríguez, L. \& Moreno, Z.(2013) Perspectivas de liderazgo consideradas por Bolman y Deal: un análisis en los gerentes venezolanos. Telos. Revista de Estudios Interdisciplinarios en Ciencias Sociales, Vol. 15, No. 1 (2013) 49 -63.

Rodríguez, D. y Opazo, P. (2007). Comunicaciones en la Organización. Ediciones Universidad Católica de Chile, Santiago.

Ruiz e Ispizúa, La descodificación de la vida cotidiana, Bilbao, Universidad Deusto, 1989.

Taylor, S.J. y Bogdan, R. (2000). Introducción a los métodos cualitativos de investigación. Ed. Paidós.

UNESCO. (2012). Directrices de la UNESCO para el reconocimiento, validez y acreditación de los resultados del aprendizaje no formal. UNESCO. Alemania.

Schmelkes, S. \& Manteca, E. (2001). Calidad de la educación y gestión escolar. En Gestión Escolar. Programa y materiales de apoyo para el estudio. SEP. México. 25-38

Sergiovanni, T. J. (1992). Moral leadership. Getting the heart of school improvement San Francisco: Jossey-Bass. 
Sergiovanni, T. J. (2001). Leadership. What's in it for schools? Londres, Routledge Falmer.

Semprum y Fue mayor. (2007). Un genuino estilo de liderazgo educativo. ¿Una realidad o una ficción institucional. Laurus, 13, 23, 350-380.

Schwartz, H. and Jerry J. (1979) Qualitative Sociology: A Method to the Madness. The Free Press. New York.

Wilber, K. (1997). Breve historia de todas las cosas. Barcelona: Kairós. Wilber, K. (2000) A theory of everything. Boston.

Wilber, K. (2010). A Summary of My Psychological Model - Or Outline of an Integral Psychology.

Wrigth, S. (2010, agosto). Perceptions are perspectives. Multi-rater assessment in integral leadership development. Conferencia presentada en Integral Theory Conference, Pleasant Hill, California.

ABDL (2004). Associacao Brazileira para o Desenvolvimiento de Lideranças: De lo posible e los nuevos posibles. Disponible en: http://www.lead.org.br/

UNESCO-IIPE- IWGE (2012). From schooling to learning. Paris: UNESCO/ International $\begin{array}{llll}\text { Institute } & \text { for } & \text { Educational }\end{array}$ http://unesdoc.unesco.org/images/0021/002192/219289e.pdf 